\title{
Earth's climate as a problem in physics
}

\author{
Franco Prodi ${ }^{1, a}$ and Alfonso Sutera ${ }^{2, b}$ \\ 1 Institute of Sciences of Atmosphere and Climate, Via Gobetti, 10140129 Bologna, Italy \\ 2 Department of Physics, University of Rome "La Sapienza", Piazzale A. Moro, 500185 Rome, Italy
}

Received: 15 May 2012

Published online: 22 May 2012 - (C) Società Italiana di Fisica / Springer-Verlag 2012

\begin{abstract}
Among the outstanding problems that physics has to face, surely, the understanding of the climate's behavior can be considered one of the most challenging. All the fundamental forces seem to operate at the lowest order. However, it appears that they operate with a large degree of randomness wherein a systematic (deterministic) behavior is floating in this sea of uncertainty. It is hard, in fact, to exclude that observations just describe the outcome of an erratic dynamics, while it is equally hard to rule out that certain effects may have well-established causes.
\end{abstract}

Recently, it has been widely discussed whether man may substantially change the climate by modifying the chemical composition of the atmosphere through the addition of gases that interact with the thermal radiation emitted by the Earth's system. In our opinion, this statement is trivially true, since a response to this forcing must surely occur. When, however, the key question is asked, i.e. how much the man-induced stress on climate will change its behavior, the answer becomes extremely gray and what is a trivial fact becomes a rather obscure subject. The reasons reside in our poor understanding of the multifold interactions that a perturbation, in a particular component of the climate system, undergoes before its effect is manifest at the macroscopic level. Moreover, while on a particular spatial-temporal scale such a perturbation may have a well-described effect, on other spatial-temporal scales it could be so diluted to become an irrelevant component of the system. The state of the affair, therefore, is an evolving subject that must be approached by a careful analysis of the possible outcomes by manifold techniques, ranging from observational studies up to attempts to simulate the full process by numerical tools.

In this focus point we present three approaches, which touch upon a few topics. We are sure that our choice is covering just a subset of open questions and other equally important aspects have been disregarded. Our aim, however, is not to be complete or inclusive of all the aspects influencing the climate's behavior. It is, instead, to present to the community of physicists the current thinking about climate and its behavior, in the hope that an open debate on this journal will start about climate as a physical system. In this endeavor we decided to ask three prominent scientists to outline their opinion and views.

By listing in alphabetic order, Reinhard Böhm - Central Institute for Meteorology and Geodynamics (ZAMG), Vienna, Austria - described trends in long time series of air pressure, air temperature and precipitations in central Europe and offered a few inferences on the regional climate variability [1].

Klaus Fraederich - KlimaCampus, Hamburg, Germany - introduced a hierarchy of global spectral circulation models ranging from the shallow-water system via the primitive equation dynamical core of the atmosphere to the Planet Simulator as a Global Climate Model (GCM) of Intermediate Complexity (MIC) [2].

Richard Lindzen - Program in Atmospheres, Oceans and Climate Massachusetts Institute of Technology, USApresented an illuminating discussion on the greenhouse effect and discussed his view about the sensitivity of climate to a perturbation to the energy budget [3].

\footnotetext{
a e-mail: f.prodi@isac.cnr.it

b e-mail: alfonso.sutera@roma1.infn.it
} 
The authors made a special effort, while presenting their work, in reaching out the widest audience of scientists including those who have not been previously exposed to climate physics and its behaviour.

We hope to have been provocative enough to stimulate the debate. We will make a point of our editorial activity that alternative views find a fair and open environment for discussion within this journal.

\section{References}

1. R. Böhm, Eur. Phys. J. Plus 127, 54 (2012).

2. K. Fraedrich, Eur. Phys. J. Plus 127, 53 (2012).

3. R.S. Lindzen, Eur. Phys. J. Plus 127, 52 (2012). 